\title{
Convenient Preparation of Superhhydrophobic Ormosil Powder
}

\author{
Baiyu $\mathrm{Li}^{\mathrm{a},{ }^{*},}$, Xiaogang Gao ${ }^{\mathrm{b}}$ and Zaiman $\mathrm{Liu}^{\mathrm{c}}$ \\ The School of Chemical and Biological Engineering, Lanzhou Jiaotong University, Lanzhou730070, \\ China

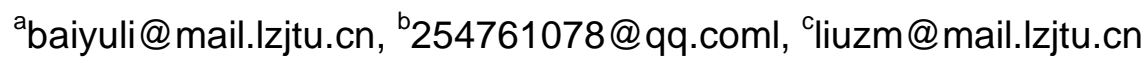

Keywords: superhydrophobic; ormosil; polymethylsilsesquioxane; powder; silica Abstract. Methyltrimethoxysilane (MTMS) was used as the precursor to prepare organically modified silica (ormosil) aerogel powder. The preparation was done in two steps. In the first step, MTMS was transformed into a gel through crosslinking by a small amount of sodium silicate, and the gel was broken into small pieces by strong shearing. In the second step, additional MTMS that was prehydrolyzed by acid catalyzed hydrolysis was added to the former gel dispersion, then ammonium hydroxide was added to make the $\mathrm{pH}$ to be about 11. An instant precipitation of white solid was observed after ammonium hydroxide addition. Superhydrophobic ormosil powder was obtained after ambient pressure drying of the precipitated solid. Scanning electron microscopy(SEM) observation shows that the obtained powder was of porous structure resembling aerogel.

\section{Introduction}

Superhydrophobic surfaces have been widely studied these years because of their versatile potential applications. For example, superhydrophobic textiles could be used to fabricate filter for oil-water separation [1]. Self-cleaning surfaces could be made by mimicking the hierarchically rough surface of natural self-cleaning plants $[2,3]$. Metal surfaces with superhydrophobicity could withstand long-term exposure to corrosive liquids, e.g. acidic, basic and salt solutions [4]. Superhydrophobic surfaces are also able to prevent ice formation upon impact of supercooled water both in laboratory conditions and in natural environments [5].

The apparent contact angle of a droplet of water on a rough surface is given in Eq. 1 [6].

$$
\cos \theta^{\prime}=r \cos \theta
$$

where $r$ is the roughness factor, which is defined as the ratio of the area of a rough surface to its geometric projected area. $\theta$ is the contact angle of water on a flat surface, and $\theta$ ' the apparent contact angle on its corresponding rough surface.

This means that a rough surface is more hydrophobic than a flat surface for an intrinsically hydrophobic material, of which $\theta>90^{\circ}$ [6]. Accordingly, surfaces are designed to be of low fraction of solid area, using materials that are intrinsically hydrophobic, the larger the $\mathrm{r}$ or $\theta$, the larger the $\theta^{\prime}$. The surface fraction of solid could be decreased by increasing the surface roughness. In general, surfaces with a static contact angle higher than $150^{\circ}$ are defined as superhydrophobic surfaces [7]. High surface roughness has been realized by a variety of ways, e.g. through template reproduction [8], phase separation [9], sol-gel [10], and electrodeposition [11] etc.

Aerogels are materials obtained when liquids in wet gels are removed with the gel network remained [12]. Therefore, aerogels are of porous structure in nature with low solid surface fraction. Silica aerogels are among the most widely studied aerogel materials. Superhydrophobicity may be realized for silica aerogels by proper modification of their surface hydrophobicity. For this purpose, organosilanes are frequently used to silylate silanol groups on surface of silica aerogels, using co-precursor [13] or post treatment [14] methods. Silica-type aerogels can also be directly prepared by 
using alkyltrialkoxysilane as the starting material, e.g. methyltrimethoxysilane (MTMS) or methyltriethoxysilane (MTES). Silica through any of these modifications is frequently referred to as ormosil (organically modified silica) $[15,16]$.

Wet gels have to be dried under specially controlled conditions, e.g. supercritical states, to overcome capillary force damage to network structure. To retain network at ambient pressure drying processes, time-exhausting solvent exchange procedures are often used, to replace the pore liquids with solvents of lower surface tensions [12].

In the present work, by using MTMS as the precursor, we have developed a rapid and convenient two-step method for the preparation of ormosil powder of superhydrophobicity. In the first step, a hydrogel of MTMS was first prepared and broken into small pieces to obtain a dispersion of hydrogel particles. Then hydrolysis and base catalyzed polycondensation of MTMS took place in the hydrogel dispersion to result in a precipitated powder. Drying this powder at ambient pressure gave a superhydrophobic ormosil powder.

\section{Experimental}

Materials. Methyltrimethoxysilane (MTMS) was purchased from Qufu Chenguang Chemical Corporation Ltd. (Shandong, China), and used as received. Hydrochloric acid (38 wt.\%), ammonium hydroxide (25 wt.\%) and sodium silicate $\left(\mathrm{Na}_{2} \mathrm{SiO}_{3} \cdot 9 \mathrm{H}_{2} \mathrm{O}\right)$ are all of analytical grade. Ammonium hydroxide was used as received, hydrochloric acid was diluted to $0.5 \mathrm{M}$ solution and sodium silicate was dissolved in water to be used in the form of $20 \mathrm{wt} \%$ solution. Deionized water was used throughout the experiments.

Preparation of ormosil powder. To $20 \mathrm{~mL}$ of water, $1 \mathrm{~mL}$ of MTMS was added and $\mathrm{pH}$ was adjusted to 3 using $0.5 \mathrm{M}$ hydrochloric acid solution. After $30 \mathrm{~min}, 1 \mathrm{~mL}$ of sodium silicate solution (20 wt.\%) was added to the hydrolyzate to act as the base catalyst for gelation to take place. It could be observed that the hydrolyzate lost flowability in about $10 \mathrm{~min}$.

Then, another $10 \mathrm{~mL}$ of hydrolyzate, which was prepared with the same method as that formerly used for gel formation catalyzed by acid, was added to the gel. After that, the gel was broken to a dispersion of small pieces of gel particles under strong shearing using a high speed homogenizer at $10,000 \mathrm{rpm}$ (FS-2, China). To this gel dispersion, additional $50 \mathrm{~mL}$ of hydrolyzate of MTMS (the same as above) was added and homogenized, and ammonium hydroxide was added to adjust the $\mathrm{pH}$ to be about 11. It could be observed that white precipitate appeared and enriched to the upper section of the liquid. The collected solid was dried in air at ambient temperature to obtain a white powder (denoted as powder A hereafter).

As a comparison, the acid catalyzed hydrolyzate of MTMS, which is the same as above, was treated by ammonium hydroxide to get $\mathrm{pH}=11$, also leading to a white precipitate, and ambient pressure drying of this precipitate also gave a white powder (denoted as powder B hereafter).

Characterization. For hydrophobicity measurement, a glass slide was coated with a thin layer of polymethylsilsesquioxane resin, which was prepared according to a literature method [17] but diluted with ethanol. After solvent evaporation, a thin layer of viscous PMSQ coating was formed. Powder samples were adhered on this glass slide by spreading a thin layer of their dispersion in ethanol. Hydrophobicity was evaluated by static water contact angle measurement, which was performed on a DSA100 contact angle analyzer (Kruss, Germany). A drop volume of $8 \mu \mathrm{L}$ was adopted for water contact angle measurements. For each sample, five measurements were made at different places to give an average result.

The microscopic morphologies of the solid powders were observed on a JSM-6701F SEM (Japan). The specimens were prepared according to the same method as that for water contact angle 
measurement except that thinner glasses were used in this case. The specimens were sprayed with gold in vacuum after drying in air for SEM observation.

\section{Results and Discussion}

The difference between MTMS and tetraethoxysilane (TEOS), which is frequently used as precursor of silica, is that MTMS can be thought to be the product when one ethoxy group in TEOS is replaced by a hydrophobic methyl group. This difference leads to less functional groups existing in the hydrolyzate of MTMS when compared with that of TEOS. As a result, it is always more difficult for the hydrolyzate of MTMS to transform into a gel. In the present work, it was found that addition of a small amount of sodium silicate facilitated the gelation process through increasing the functionality of the system. Sodium silicate acted as both crosslinker and base catalyst to transform the hydrolyzate of MTMS into a gel in quite short a time of several minutes.

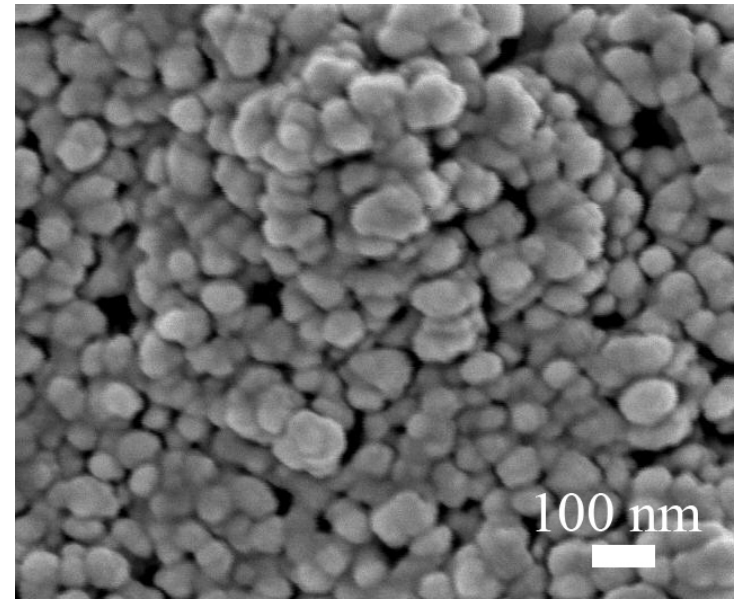

Fig.1 SEM image of powder A from two-step method

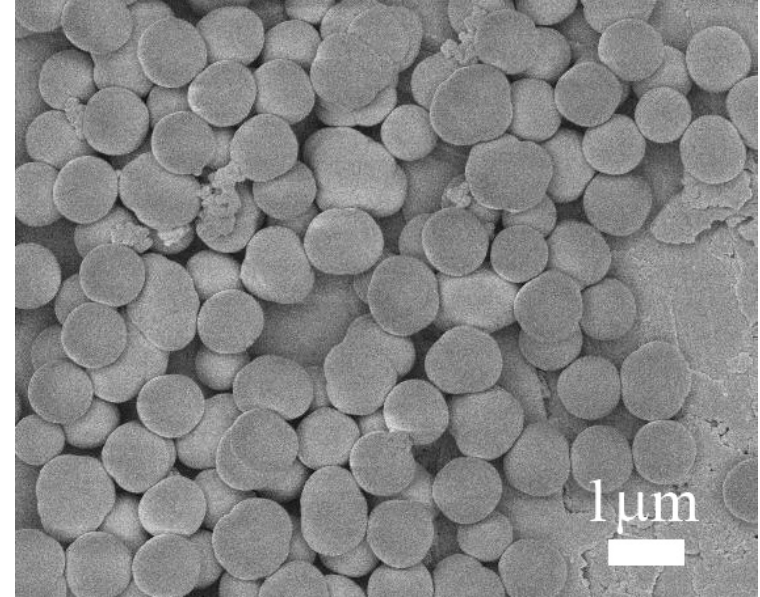

Fig.2 SEM image of powder B from one-step method

Fig. 1 shows the SEM image of sample A, which is an ormosil powder prepared by the two-step method. Fig. 2 shows the SEM image of sample B, which was prepared by the one-step method. Interestingly, it is found that sample A is of porous structure, consisting of interconnected small particles of about $100 \mathrm{~nm}$ in size. While sample B is smooth spheres of micron size. In the case of the two-step method, polycondensation reaction of the hydrolyzed MTMS took place in between the small pores of small hydrogel particles, taking advantage of the network of hydrogel as nucleation center to grow rapidly to form small particles. While in the case of the one-step process, the oligomeric hydrolyzate of MTMS aggregated to form small nucleation centers, and particles grew through followed addition of smaller particles to these centers. The formed particles are much larger because of the lack of growing centers [18]. In the drying process of sample A, the small particles interconnected by gel framework are stiff enough to survive severe capillary force without much damage to network structure, retaining porous structure.

The static water contact angle measurement for sample A gives an average value of $155.26 \pm 5.25^{\circ}$, which is in the superhydrophobic range, while that for its counterpart sample B is $136.2 \pm 0.9^{\circ}$. The higher water contact angle of sample $\mathrm{A}$ is attributed to its porous structure, which gives a much higher $\mathrm{r}$ in Eq. 1. 


\section{Conclusions}

It was found to be difficult for the acid catalyzed hydrolyzate of MTMS to form a gel in aqueous medium. Addition of a small amount of sodium silicate solution to the hydrolyzate increased the number of functional groups and lead to rapid gel formation, while simple base treatment with ammonium hydroxide lead to formation of smooth spheres of ormosil rather than a gel.

Base catalyzed polycondensation of MTMS hydrolyzate in the dispersion of small pieces of broken gel in water resulted in rapid formation of a white precipitate. The obtained ormosil powder after drying the precipitate at ambient pressure shows aerogel-like porous structure in SEM image. This means that nucleation and growth took place in the network of the gel, taking advantage of the gel framework as the nucleation center, leading to porous structure consisting of interconnected ormosil particles about $100 \mathrm{~nm}$ in size. Water contact angle measurements show that the obtained porous ormosil powder is superhydrophobic, due to its highly porous structure.

Because the gel formation and polycondensation in dispersion of broken gel are all rapid processes, and the drying process is carried out at ambient pressure, this method is convenient to use for the practical production of superhydrophobic ormosil powder.

\section{References}

[1] C.H. Xue, P.T.Ji, Hanraads, P. Zhang, Y.R. Li, and S.T. Jia, Appl. Surf. Sci. 284 (2013) 464-471.

[2] B. Bhushan, K. Koch, and Y.C. Jung, Appl. Phys. Lett. 93(2008) 093101-1-093101-3.

[3] B. Bhushan, Y.C. Jung, and K. Koch, Langmuir 25 (2009) 3240-3248.

[4] M.N. Qu, B.W. Zhang, S.Y. Song, L. Chen, J.Y. Zhang, and X.P. Cao, Adv. Funct. Mater. 17 (2007) $593-596$.

[5] L.L. Cao, A.K. Jones,V.K. Sikka,J.Z. Wu, and D. Gao, Langmuir 25 (2009) 12444-12448.

[6] S. Shibuichi, T. Onda, N. Satoh, and K. Tsujii, J. Phys. Chem. 100 (1996) 100, 19512-19517.

[7] S.T. Wang, L. Jiang, Adv. Mater. 19 (2007) 3423-3424.

[8] M.P. Spellings, T.J. Cooksey, and G.K. Jennings, Macromol. Rapid Commun. 35 (2014) 1937-1942.

[9] P. A. Levkin, F. Svec, and J. M. J. Fréchet, Adv. Funct. Mater. 19 (2009) 1993-1998.

[10] G. Zhang, D.Y. Wang, Z.Z. Gu, and H. Möhwald, Langmuir 21 (2005) 21, 9143-9148.

[11] Z. Chen, F. Li, L.M. Hao, A.Q. Chen, and Y.C. Kong, Appl. surf. Sci. 258 (2011) 1395-1398.

[12] N. Hüsing, U. Schubert, Angew. Chem. Int. Ed. 37 (1998) 22 - 45.

[13] M.D.F. Júlio, L.M. Ilharco, Microporous Mesoporous Mater. 199 (2014) 29-39.

[14] J.J. Zhu, J.M. Xie, X.M. Lü, and D.L. Jiang, Colloids Surf. A 342 (2009) 97-101.

[15] H. Budunoglu, A. Yildirim, M.O. Guler, and M. Bayindir, ACS Appl. Surf. Interfaces, 3 (2011) 539-545.

[16] J.W. Park, J.S. Kim, T.J. Park, E.H. Kim, and S.M. Koo, J. Colloid Interface Sc. 438 ( 2015) 220-226.

[17] J.K. Lee, K. Char, H.W. Rhee, H.W. Ro, D.Y. Yoo, and D.Y. Yoon, Polymer 42 (2001) 9085-9089.

[18] A. Arkhireeva, J.N. Hay, J. Mater. Chem. 13 (2003) 3122-3127. 